\title{
Non-uniformity of cross-beam laser power distribution as a source of errors in non-linear spectroscopy
}

\author{
S.E. Zelensky \\ Optics Division, Physics Department, Kyiv National Taras Shevchenko University, 6 Glushkova, 03680 Kyiv, Ukraine \\ Phone: +38(044) 266 2295; e-mail:zele@phys.univ.kiev.ua
}

\begin{abstract}
We investigated errors arising in calculations of optical signals in non-linear laser spectroscopy due to neglection of non-uniformity in cross-beam laser power distribution. The following three cases of non-linear emission are considered: saturated molecular luminescence, sequential multi-photon excitation of luminescence, laser-induced incandescence of micro-particles.
\end{abstract}

Keywords: non-uniformity of laser power spatial distribution, non-linear processes, luminescence, laser-induced incandescence.

Paper received 23.05.03; accepted for publication 17.06.03.

It is a frequent occasion in laser spectroscopy when laser radiation induces emission of an investigated object with a non-linear response on the excitation laser power. The non-linear emission can be observed, for example, in the cases of multi-photon or multi-step absorption, saturated luminescence, laser-induced incandescence of micron-sized particles, etc. Such conditions require an experimentalist to be especially careful with data processing as far as the non-uniform distribution of laser power within the investigated volume can cause significant additional uncertainties in the calculated results. The present paper considers errors caused by the use of rectangular-function approximation of non-uniform cross-beam distribution of laser intensity as compared with Gaussian-function approximation.

Consider a hypothetical laser-induced non-linear process followed by optical emission. Denote the emission power from a unit volume as $i$ and the laser surface power density as $F$. For a moderate deviation of $F$, the nonlinear dependence $i(F)$ can be approximated as

$$
i=\text { const } \times F^{\gamma} \text {, }
$$

where $\gamma$ is a convenient phenomenological measure of non-linearity of the process investigated. The value of $\gamma$ can be easily calculated from experimental data $i(F)$ pre- sented in a log-log scale. By taking logarithm of (1), $\lg i=$ $=$ const $+\gamma \mathrm{g} F$, the value of $\gamma$ can be calculated as a slope of the experimental curve $\lg i$ vs $\lg F$ :

$\gamma=\frac{d(\lg i)}{d(\lg F)}=\frac{d i / i}{d F / F}$

Obviously, for a linear process, $\gamma=1$. For a process of $N$-photon excitation of luminescence, $\gamma=N$. The informational validity of $\gamma$ increases when it takes noninteger values, for example, in the case of laser-induced incandescence. It should be emphasized, the introduced into equation (2) parameter $\gamma$ can be calculated from the experimental data regardless the photodetectors' sensitivity, transmittance of optical registration channels, geometrical arrangement of the investigated object relatively to the collective aperture, etc. That is, to calculate $\gamma$, the measured values of $i$ and $F$ can be taken in any suitable arbitrary units.

In calculations, real laser beams are often presented as equivalent beams with uniform distribution of surface power density across the beam section, i.e. $F=F_{0}$ at $r<r_{0}$ and $F=0$ at $r>r_{0}$, where $r_{0}$ is the beam radius, and $r$ is the distance from the beam axis. Hereinafter, such beams are referred to as rectangular beams. 


\section{S.E. Zelensky: Non-uniformity of cross-beam laser power ...}

In many cases, cross-beam laser intensity distribution can be approximated by Gaussian function, for example, as follows

$$
F=F_{0} \exp \left(-r^{2} / \Delta^{2}\right) \exp (-\kappa z)
$$

where $F_{0}$ is the laser power density in the beam axis at the entrance of the investigated object, $\Delta$ is the beam radius at the level of $e^{-1}, \kappa$ is the extinction coefficient at a wavelength of laser radiation, $z$ is the coordinate along the beam axis.

The experimentally measured is usually the integral emission power, hereinafter denoted as $I$, which can be calculated by integration of $i$ over the laser-irradiated volume. For Gaussian beam , the following expression can be derived

$$
\begin{aligned}
& I=\mathrm{const} \int_{0}^{\infty} 2 \pi r d r \int_{0}^{L} d z\left[F_{0} \exp \left(-r^{2} / \Delta^{2}\right) \exp (-\kappa z)\right]^{\gamma}= \\
& =\operatorname{const} F_{0}^{\gamma} \frac{[1-\exp (-\gamma \kappa L)] \pi \Delta^{2}}{\gamma^{2} \kappa}
\end{aligned}
$$

where $L$ is the length of the laser-irradiated track. As is seen from (4), the non-uniformity of spatial distribution of $F$ does not affect the power law $I=$ const $\times F_{0}^{\gamma}$. However, the radius of equivalent rectangular beam is changed to $\Delta / \sqrt{\gamma}$ and the effective length becomes [1$-\exp (-\gamma \kappa L)](\gamma \kappa)^{-1}$. Thus, on condition $\gamma=$ const, the use of rectangular-beam approximation does not cause additional errors of the experimentally measured value of $\gamma$.

It should be noted, equation (4) is valid only if $\gamma$ does not depend on the laser power density, $\gamma \neq \gamma(F)$. However, there are a lot of processes, in which $\gamma$ changes with $F$. Thereinafter, in terms of three examples of laser-induced processes with variable $\gamma$, we consider the errors caused by the use of rectangular-beam approximation as compared with Gaussian approximation.

1. With a conventional model of laser-induced molecular luminescence and a rectangular-beam approximation, on the basis of population balance equations, the luminescence power emitted from a unit volume can be written $[1]$ :

$$
i=\text { const } \frac{\Phi}{1+\Phi}
$$

where $\Phi$ is the dimensionless laser intensity, $\Phi=\sigma \tau F, \sigma$ is the absorption cross-section, $\tau$ is the luminescence lifetime. Equation (5) involves a well-known phenomenon of luminescence saturation. For Gaussian approximation (3), in case of low absorption of laser radiation, $\kappa<<1 / L$, the integration of (5) over the volume gives the following expression for the integral luminescence power

$$
I=\mathrm{const} \cdot \ln \left(1+\Phi_{0}\right)
$$

where $\Phi_{0}=\sigma \tau F_{0}$. The constants in (5) and (6) depend on the sensitivity of photodetectors and on the geometrical arrangement of the experiment. To eliminate these unknown constants, the following dimensionless parameter can be introduced. The relative yield of luminescence, defined as the ratio of the saturated luminescence power to the non-saturated luminescence power at the same level of laser excitation, is hereinafter denoted as $\eta$. With the use of (5) and (6), the following expressions can be derived: for rectangular beam approximation

$\eta_{R}=\frac{1}{1+\Phi_{0}}=\gamma_{R}$

and for Gaussian approximation

$\eta_{G}=\frac{\ln \left(1+\Phi_{0}\right)}{\Phi_{0}} ; \gamma_{G}=\frac{\Phi_{0}}{\left(1+\Phi_{0}\right) \ln \left(1+\Phi_{0}\right)}$

As is seen from (7) and (8), at high levels of laser excitation, the well-known laser-induced luminescence is a process with variable $\gamma$. The appropriate plots are given in Fig. 1a.

With the use of experimental data $\eta\left(F_{0}\right)$ and $\chi\left(F_{0}\right)$, one can determine the parameters $\sigma \tau$ of the investigated molecules. As is seen from Fig. 1a, the use of rectangular-beam approximation causes significant errors as com-
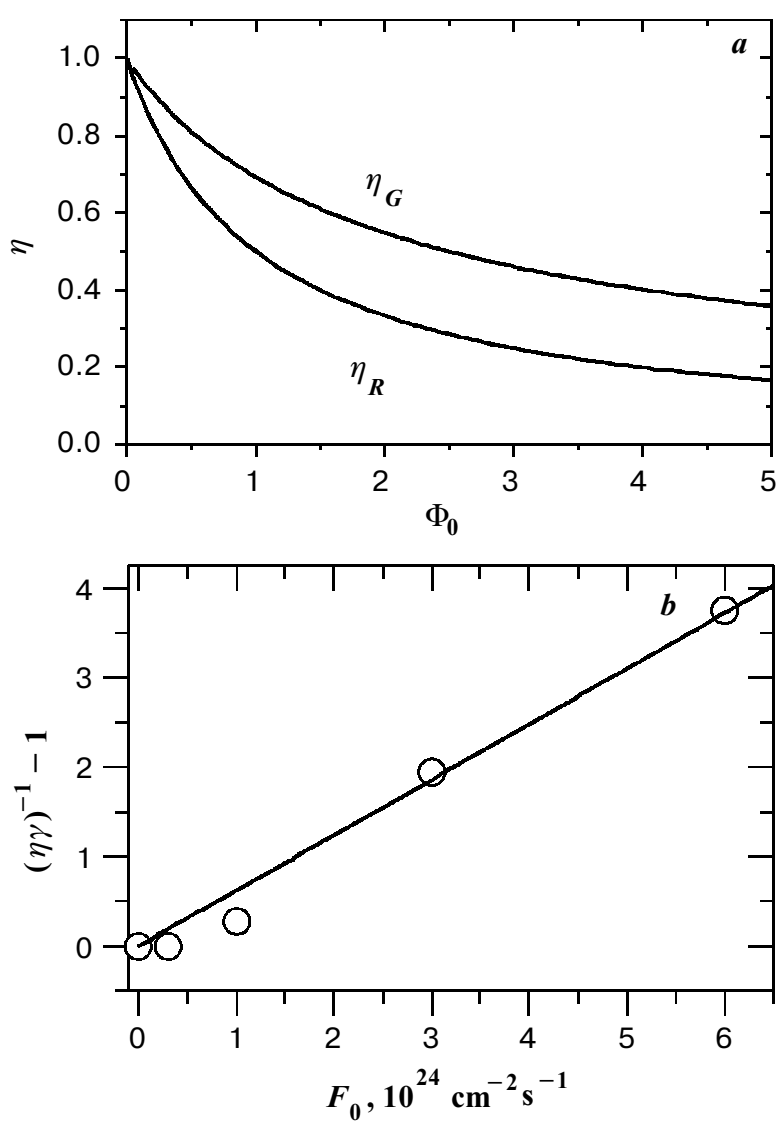

Fig. 1. Calculated (solid lines) and experimental (circle points) plots of $\eta$ and $\gamma$ for laser-induced luminescence of Coumarin dye solution. 


\section{S.E. Zelensky: Non-uniformity of cross-beam laser power ...}

pared with Gaussian approximation. For example, if the experiment shows the two-fold saturation of luminescence $(\eta=0.5)$, then the value of $\sigma \tau$ obtained with the use of rectangular-beam approximation is underestimated by a factor of 2.5 as compared with Gaussian approximation.

Besides, the following circumstance is worth noting. According to (7) and (8), the values of $\eta$ and $\gamma$ coincide in case of rectangular beam and are essentially different $\left(\gamma_{G}>\eta_{G}\right)$ in case of Gaussian approximation. The experiments confirm this feature. Fig. $1 b$ presents the experimental data of $\eta$ and $\gamma$ for an aqueous solution of Coumarin-120 dye excited by a Q-switched YAG-Nd ${ }^{3+}$ laser (third harmonic wavelength $355 \mathrm{~nm}$, pulse duration $25 \mathrm{~ns})$. The luminescence was detected at a fixed wavelength. According to (8), the experimental data plotted in Fig. $1 \mathrm{~b}$ can be approximated with the following linear function, $\left(\eta_{G} \gamma_{G}\right)^{-1}=1+\sigma \tau F_{0}$, and the following estimate can be made $\sigma \tau=6 \cdot 10^{-25} \mathrm{~cm}^{2} \mathrm{~s}$.

2. Consider one more example of a non-linear process with variable $\gamma$. As is shown in [2-4], $\mathrm{Nd}^{3+}$ ions in YAG crystals demonstrate luminescence from high energy levels, for example, from ${ }^{2} \mathrm{~F} 2{ }_{5 / 2}$ metastable level, which lies at a distance of $38000 \mathrm{~cm}^{-1}$ from the lowest level ${ }^{4} \mathrm{I}_{9 / 2}$. When the crystal is excited with a pulsed laser radiation at a wavelength of $532 \mathrm{~nm}$ (second harmonic of YAG$\mathrm{Nd}^{3+}$ laser), level ${ }^{2} \mathrm{~F} 25 / 2$ is populated by sequential absorption of laser photons. With the use of a Q-switched laser with pulse duration of $25 \mathrm{~ns}$, the experiments show that the luminescence from ${ }^{2} \mathrm{~F} 2_{5 / 2}$ level is characterized with $\gamma$ varying from 2 to 3 depending on the excitation intensity. Note should be made, $\gamma$ increases with $F$, which can be approximated as follows

$i=C_{1} F^{2}+C_{2} F^{3}$

where $C_{1}$ and $C_{2}$ are the constants. Obviously, in case of rectangular-beam approximation, for spatially integrated luminescence, the dependence $I(F)$ will be similar to (9). For Gaussian approximation (3), with taking account for (4), the integral luminescence power can be written

$I=\pi \Delta^{2} L\left(\frac{1}{2} C_{1} F_{0}^{2}+\frac{1}{3} C_{2} F_{0}^{3}\right)$

Fig. 2 presents the plots of $\gamma$ calculated with the use of (9) and (10). As is seen from the plots, the values of $F$, at which the three-step absorption overpowers the two-step absorption, differ by a factor of 1.5 for a rectangularbeam and Gaussian approximation, hence the errors increase.

3. It is observed experimentally [5-10] that the radiation of Q-switched lasers can overheat light-absorbing micronsized particles in gases and in condensed matter to a temperature of thousands of Kelvins, hence the laser-heated particles emit easily-detectable thermal radiation. Consider a simplified model of laser-induced incandescence

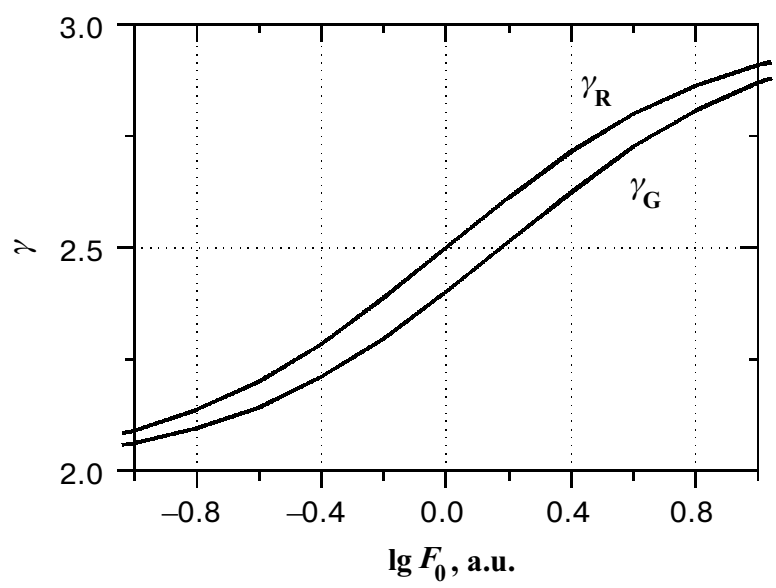

Fig. 2. Calculated plots of $\gamma$ as a function of laser power surface density for laser-induced luminescence of an YAG: $\mathrm{Nd}^{3+}$ crystal.

of suspended spherical black-body particles in transparent condensed matter. The numerical treatment [9] of heat balance equation shows, that, at a first approximation, during the action of laser pulse, temperature of a laserheated particle, $T$, is proportional to the laser surface power density, $F$, that can be written

$T=\xi F$

where $\xi$ is the constant. Then the dependence of power of thermal radiation, $i$, at a fixed wavelength, $\lambda$, on the laser power density can be derived by substituting equation (11) into the Planck law of black-body emission. This yields

$i=\frac{\text { const }}{\exp \left(h c / k_{B} \lambda \xi F\right)-1}$

where $h$ is the Planck constant, $k_{B}$ is the Boltzmann constant, $c$ is the speed of light. According to definition (2), from (12) the following can be derived

$\gamma=\frac{h c / k_{B} \lambda \xi F}{1-\exp \left(h c /-k_{B} \lambda \xi F\right)}$

So, the laser-induced incandescence of suspended particles in condensed matter is a process with variable $\gamma$. As is seen from (13), the value of $\gamma$ significantly changes with $F$. Fig. 3 presents the results of measurement of $\gamma$ at a wavelength of $500 \mathrm{~nm}$ in the aqueous suspension of submicron black particles excited by a Q-switched YAG$\mathrm{Nd}^{3+}$ laser (excitation wavelength $1064 \mathrm{~nm}$ ). The following distinctive feature of laser-induced incandescence should be noted: $\gamma$ undergoes a significant decrease (from 5 to 2) at moderate variation of $F$ (from 5 to $10 \mathrm{MW} \mathrm{cm}^{-2}$ ).

With regard for (11), equation (13) can be considered as a method for determination of particle temperature using an experimentally measured $\gamma$. However, seeing the strong dependence of $\gamma$ on $F$, we can expect signifi- 


\section{S.E. Zelensky: Non-uniformity of cross-beam laser power ...}

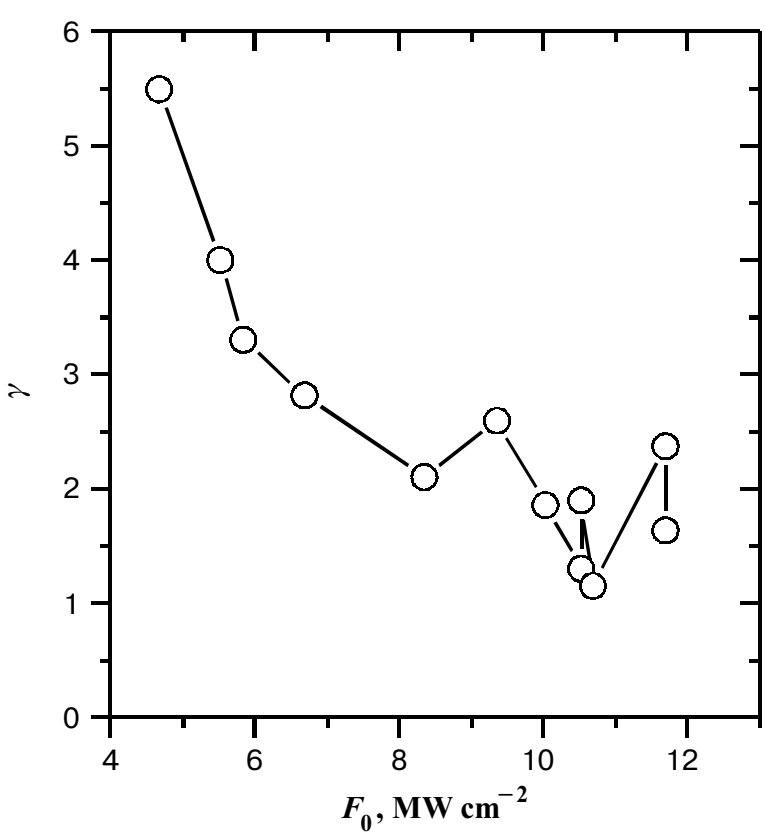

Fig. 3. Parameter $\gamma$ as a function of laser power surface density for laser-induced incandescence of an aqueous suspension of light-absorbing submicron particles.

cant errors caused by the disregard of non-uniform distribution of laser power density. For example, the appropriate calculations performed in [10] show that the estimates of particle temperature, based on rectangular-beam and Gaussian approximation, are respectively $9000 \mathrm{~K}$ and $5600 \mathrm{~K}$ at $F=7 \mathrm{MW} \mathrm{cm}^{-2}$.

To conclude, the following can be inferred: for nonlinear processes with variable parameters $\gamma$, the neglect of non-uniformity of laser power cross-beam distribution causes significant errors in estimations of characteristics of the investigated processes.

\section{References}

1. Bely M.U., Zelensky S.E., Okhrimenko B.A. On the errors in luminescent analysis caused by the violation of a preset field approximation // Ukrainian Phys. Journ. 37(7), pp. 994-998 (1992).

2. Quarles G.J., Venikouas G.E., Powell R.C. Sequential twophoton excitation processes of $\mathrm{Nd}^{3+}$ ions in solids // Phys. Rev. B. 31(11), pp. 6935-6940 (1985).

3. Bely M.U., Grabovsky V.V., Zelensky S.E., Okhrimenko B.A. Competition of two-step and three-step transitions in YAG: $\mathrm{Nd}^{3+} / /$ Quant. Electron. (Kiev). 38, pp. 24-28 (1990).

4. Bely M.U., Grabovsky V.V., Zelensky S.E., Naumenko S.N., Okhrimenko B.A. Multi-step optical transitions in YAG: $\mathrm{Nd}^{3+}$ at intense excitation // Sov. Journ. Appl. Spectrosc. 52(1), pp. 118-121 (1990).

5. Cignoli F., Benecchi S., Zizak G. Time-delayed detection of laser-induced incandescence for the two-dimensional visualization of soot in flames // Appl. Opt. 33(24), pp. 5778-5782 (1994).

6. Vander Wal R.L., Weiland K.J. Laser-induced incandescence: Development and characterization towards a measurement of soot-volume fraction // Appl. Phys.B. 59, pp. 445452 (1994).

7. Vander Wal R.L. Laser-induced incandescence: detection issues // Appl. Opt. 35(33), pp. 6548-6559 (1996).

8. McManus K.R., Frank J.H., Allen M.G., Rawlins W.T. Characterization of laser-heated soot particles using optical pyrometry // Amer. Inst. Aeronaut. and Astronaut. AIAA-980159. 36-th Aerospace Sciences Meeting and Exhibit, Jan.1215, 1998. Reno, NV, pp. 1-8.

9. Zelensky S. Laser-induced heat radiation in borate glass // J.Phys.: Condens.Matter 10, pp. 7267-7272 (1998).

10. Zelensky S. Laser-induced heat radiation of suspended particles: a method for temperature estimation // J. Opt. A: Pure Appl. Opt. 1, pp. 454-458 (1999). 